\title{
Incoherencias en la práctica de la entrevista motivacional.
}

\section{Incoherences in motivational interviewing practice.}

\section{resumen/abstract:}

El interés, la formación y la práctica de la entrevista motivacional en el ámbito de las adicciones han experimentado un desarrollo espectacular en las últimas décadas. Este impulso, sin duda positivo, convive, no obstante, con conceptos con los que es incoherente. Por un lado, con la visión del comportamiento adictivo como irracional o patológico (modelo de enfermedad más o menos 'cerebral'); por otro, con una visión de la motivación como estado interno de naturaleza cognitiva previo a la acción., fuertemente vinculada a la popularización del modelo de las 'fases' del cambio. Frente a este planteamiento, la estrategia de la validación, como elemento central y definitorio de la entrevista motivacional, requiere asumir la racionalidad del comportamiento adictivo y de las resistencias a su abandono, así como el carácter contextual y no internalista de la disposición al cambio. En particular, se cuestiona el modelo de las 'fases' y se defiende una visión de la disposición al cambio no como previa y causante de la acción, sino como resultado de la relación entre la acción y sus resultados, señalando sus implicaciones para la práctica clínica.

The interest, training and practice of motivational interviewing in the field of addictions have experienced a spectacular development in recent decades. This impulse, undoubtedly positive, coexists, nevertheless, with some concepts which are inconsistent. On the one hand, with the vision of addictive behaviours as irrational or pathological (more or less in line with the Brain Disease Model of Addiction); on the other, with the vision of motivation as an internal state of cognitive nature that precedes action and is strongly linked to the popular model of Stages of Change. Faced with this approach, the strategy of validation, as the central and defining element of the motivational interview, requires assuming the rationality of the addictive behaviour and the resistance to its abandonment, as well as the contextual and non-internalism nature of the willingness to change.

In particular, the Stages of Change model is questioned and a vision of attitudes towards change is defended not as prior or causal to the action but as a result of the relation between actions and its results, highlighting its implications for clinical practice.

\section{palabras clave/keywords:}

Adicción, tratamiento, entrevista motivacional, contextualismo, acción, modelo de fases de cambio.

Addiction, treatment, motivational interviewing, contextualism, action, stages of change model. 
No es apropiado pensar que la Entrevista Motivacional sea una técnica o un conjunto de técnicas que se aplican o (peor aún) se 'utilizan' con la gente...

(Rollnick y Miller, 1996)

Desde que, en 1983, el psicólogo William R. Miller publicó su primer texto sobre entrevista motivacional (Miller, 1983) el desarrollo de esta estrategia ha sido espectacular. En un artículo publicado en 2009, Miller y Rollnick (2009) calculaban que, desde entonces, el número de publicaciones sobre entrevista motivacional se había ido duplicando cada tres años, y el número de profesionales formados hasta ese momento superaba los 15 millones. Desde hace al menos dos décadas, es raro encontrar en nuestro país alguna jornada, congreso o reunión profesional en el ámbito de las conductas adictivas en cuyo programa no se incluya alguna comunicación, mesa redonda o taller formativo sobre esta cuestión. Además, este crecimiento cuantitativo ha ido acompañado de la generalización de la aplicación de la entrevista motivacional fuera del ámbito de las adicciones, hacia prácticamente cualquier campo en el que el profesional se enfrenta a la tarea de promover cambios de hábitos relacionados con la salud, como la alimentación, la actividad física, el comportamiento sexual, la salud bucodental, la depresión, el seguimiento de prescripciones u otros.

Ciertamente, cambiar hábitos y, en particular, conseguir el abandono de un comportamiento adictivo, no es tarea fácil. Exige disponer de tratamientos con evidencia de efectividad, pero, sobre todo, el compromiso y la disposición del propio consumidor para acometer un proceso de aprendizaje complejo que suele implicar renuncias, malestares, vacilaciones y aparentes retrocesos. De nada sirve un tratamiento eficaz si su beneficiario potencial no está dispuesto a utilizarlo, o si parte de expectativas poco realistas que tienden, por definición, a no cumplirse, generando frustración y abandono. Pensemos, además, que buena parte de la tarea de los profesionales se dirige a promover de modo oportunista el abandono del consumo de tabaco, cannabis, alcohol u otras drogas con personas que acceden a las consultas por otros problemas de salud diferentes, sin la intención inicial de abordar el problema de estos consumos y sin ninguna disposición previa para su abandono.

La incorporación exitosa de la entrevista motivacional al repertorio de destrezas profesionales en el ámbito de la clínica de las adicciones es, por lo tanto, fácil de comprender, porque se dirige a cubrir una necesidad percibida a diario por los profesionales que se dedican a estas tareas y que se encuentran con la resistencia, de parte de los consultantes, a 'convencerse' y a seguir las bienintencionadas e irreprochables advertencias, consejos, palmadas en el hombro o broncas que, según los casos y el momento, administran a sus pacientes consumidores de tabaco u otras sustancias. Existe evidencia sólida que apoya el uso de la entrevista motivacional junto con la terapia cognitivo conductual en la clínica de las adicciones (Becker et al., 2013; Comité Nacional para la Prevención del Tabaquismo, 2015; Davis et al., 2015; Gates, Sabioni, Copeland, Le Foll y Gowing, 2016; Lee et al., 2014;).

Esta incorporación creciente de la entrevista motivacional a la práctica clínica es, sin duda, algo de lo que debemos felicitarnos. No obstante, tal vez porque la mayor parte de quienes la practican no son (ni tienen por qué ser) profesionales de la psicología, o tal vez por la 
escasa relevancia que suele concederse a esta cuestión en los programas de formación, esta práctica suele estar reducida a la mera aplicación de una serie de técnicas (o 'trucos'), o suele estar vinculada a visiones insuficientes o incoherentes sobre el cambio de comportamiento, lo cual limita su efectividad.

Es importante destacar que en las revisiones sobre la efectividad de la entrevista motivacional realizadas por Hettema, Steele y Miller (2008) y por Lundahl, Kunz, Brownell, Tollefson y Burke (2010) se concluye que cuando la aplicación está guiada por un manual, donde se especifica meramente una secuencia de procedimientos, se obtienen peores resultados que cuando está guiada por principios teóricos y estrategias más generales. Además, el propio acercamiento centrado en el cliente que caracteriza a la entrevista motivacional hace contradictorio el uso de un protocolo cerrado de procedimientos. Como apuntan los propios Rollnick y Miller en la cita que abre este artículo, la entrevista motivacional no es un mero conjunto de técnicas o 'trucos' que podamos añadir sin más a nuestro repertorio sin tocar para nada al resto y sin afectar al fundamento teórico de lo que hacemos. Por el contrario, la entrevista motivacional está vinculada a una manera de entender el comportamiento y su modificación, que nos obliga, a menudo, a un cambio de perspectiva. De esa visión diferente puede derivarse, además (y esto es lo relevante), un enriquecimiento de las estrategias a poner en marcha en orden a mejorar la permeabilidad a nuestra influencia y la disposición al cambio por parte de nuestros consultantes. Este artículo pretende llamar la atención sobre algunos de estos conceptos.

\section{La entrevista motivacional es coherente con un modelo contextualista de la adicción}

Ante todo, la práctica coherente de la entrevista motivacional asume, frente al reduccionismo fisiologicista subyacente al concepto de 'enfermedad', tan alentado por la industria farmacéutica, que el comportamiento adictivo, como toda conducta, se gesta, se mantiene y se explica por su función instrumental a partir de la transacción biográfica del organismo con los contextos. Un desarrollo extenso de este modelo puede encontrarse en Costa y López (2008), y López y Costa (2014) y, en lo referido al comportamiento de fumar, en Costa y Moreno (2008).

Fumar es un comportamiento que aparece típicamente durante la adolescencia, y como resultado de la interacción entre determinadas necesidades evolutivas propias de esta etapa (curiosidad, experimentación, búsqueda de sensaciones, rebelión, búsqueda de identidad $\mathrm{y}$, especialmente, necesidad de aceptación y pertenencia al grupo de iguales) y un contexto que lo facilita (modelo de los padres o amigos, valores sociales, publicidad, accesibilidad y fácil manejo y, sobre todo, presión ejercida por el grupo). En muchos casos el consumo no pasa de ser experimental o de prueba, y es abandonado rápidamente. En otros, como sabemos, se progresa hacia un consumo ocasional, posteriormente habitual y, en poco tiempo, dependiente.

Una vez que el consumo se ha convertido en habitual, tiende a mantenerse, resultando difícil su abandono y existiendo una fuerte tendencia a la recaída si el abandono se produce. Esta situación, a la que llamamos dependencia, se produce por el valor instrumental potente 
y generalizado que adquiere la conducta de fumar, junto con su presencia constante en prácticamente todos los ámbitos de la vida del individuo.

Por lo tanto, un paradigma explicativo básico es el condicionamiento instrumental: fumar tabaco se convierte para el fumador en una herramienta sumamente útil, difícil de sustituir, segura y eficaz para conseguir efectos con un enorme valor adaptativo. Entre los reforzadores negativos del consumo está el escape o la evitación de las sensaciones aversivas producidas por la propia deprivación de nicotina, la reducción del estrés, el alivio del aburrimiento o la evitación de la ganancia de peso. Entre los reforzadores positivos está la activación o estimulación en momentos en que se requiere, la facilitación del contacto social o la integración grupal (de enorme importancia como reforzador del consumo entre adolescentes), o la mejora de la concentración y la consecuente realización de determinadas tareas que la requieren. El conocimiento de los riesgos puede tener un escaso efecto motivacional, porque se trata de consecuencias a más largo plazo y no seguras y, por lo tanto, difícilmente pueden competir con las ventajas, inmediatas y tangibles, de seguir fumando.

Por otro lado, y a diferencia de lo que sucede con otras adicciones, fumar tabaco es una conducta compatible con la mayoría de las actividades cotidianas. Los cigarrillos son ligeros, fáciles de transportar, sencillos de utilizar y tolerados socialmente. Esto hace posible que un fumador medio consuma en torno a 20 cigarrillos diarios ('un paquete'), lo que traducido a caladas, nos lleva a un consumo de 200 o más 'dosis' de nicotina cada día, consumo que se mantiene durante la mayor parte de la vida del fumador. La intensidad del consumo es, ciertamente, apabullante. Más aún: este consumo se asocia fuertemente a numerosas situaciones diarias que, de este modo, se convierten en estímulos o disparadores cuya presencia desencadena en el organismo del fumador el conjunto de respuestas fisiológicas que subjetivamente éste vive como 'deseo de fumar', según el paradigma de condicionamiento clásico o pavloviano. Obviamente, cada vez que el individuo fuma ante estos estímulos o 'disparadores' se refuerza la asociación entre éstos y la conducta de fumar. Fumar, pues, propicia un contexto favorable para seguir fumando.

Este conjunto de condicionantes que explican la conducta de fumar se complica, además, por la implicación de determinadas variables disposicionales, del individuo y del contexto, que igualmente afectan a la probabilidad del fenómeno de la dependencia. Así, la conducta de fumar está condicionada o se ve favorecida igualmente por otros aspectos del patrimonio biográfico, constituido a lo largo de la historia de aprendizaje individual, que incluye determinadas ideas o expectativas adquiridas verbalmente en el contexto social (sobre la nocividad del tabaco, sobre la propia capacidad para abandonarlo, sobre la dificultad de dejar de fumar, sobre el papel de los aspectos emocionales...), así como por el conjunto de competencias personales (para afrontar problemas, para manejar situaciones sociales, para resistir la presión del grupo, etc.) y condicionamientos de todo tipo, resultado todo ello del devenir biográfico y, en buena parte, de la propia conducta de fumar.

Así pues, fumar es una conducta morfológicamente simple pero funcionalmente compleja, y que puede llegar a impregnar la práctica totalidad de los ámbitos de la vida del fumador. 
Su modificación requiere del individuo un proceso de aprendizaje que suele ser costoso porque implica pérdidas y renuncias. Es ingenuo suponer que la mera advertencia o información sobre los riesgos sea suficiente para que los usuarios adopten una decisión y un compromiso firmes y mantenidos.

\section{Las resistencias y ambivalencias son legítimas y racionales}

A partir del modelo bosquejado en el epígrafe anterior, se comprende que las resistencias, ambivalencias, dudas, temores u objeciones que plantea el consultante fumador ante las advertencias o consejos del profesional de la salud no son el resultado de obstinación, irracionalidad, rasgos de personalidad, psicopatología o síntomas de algún tipo de 'enfermedad cerebral'. Por el contrario, la entrevista motivacional asume que estos comportamientos son legítimos, racionales y plenos de sentido.

El desafío que se plantea al fumador es dejar de utilizar un instrumento valioso que ha cumplido de modo eficaz una serie importante de funciones en el afrontamiento de situaciones cotidianas de la vida, tal como exponíamos en el apartado anterior. Numerosos fumadores se refieren al tabaco como 'un amigo', como algo que se echará de menos si se deja porque servía de apoyo, estímulo o consuelo ante distintas situaciones. Más allá de la objetividad científica que nos muestra sus indudables efectos nocivos, y más allá del irreprochable intento del profesional por promover su abandono, el resultado de esta valoración por parte del fumador puede ser la percepción de que el abandono del consumo de tabaco no compensa. Es importante no solo respetar, validar y no cuestionar de modo estratégico esta perspectiva, sino asumirla como comprensible y legítima.

Otra fuente de resistencias al cambio, más frecuente incluso, proviene de la falta de confianza en la capacidad para alcanzarlo. La llamada 'percepción de autoeficacia' es un poderoso condicionante de las decisiones (o indecisiones) de acometer tareas que requieren un esfuerzo y un coste. Nos sentimos predispuestos a iniciar un proceso costoso cuando nos percibimos capaces de conseguir nuestro objetivo, con independencia de las ventajas y beneficios, tal vez indudables, que anticipamos por su consecución. ¿Para qué esforzarme y pasarlo mal, si anticipo que no voy a conseguir lo que pretendo? Esta desconfianza en las propias capacidades es, una vez más, racional, no es una excusa ni síntoma de ninguna patología. Tampoco es algo que pueda corregirse solo con hábiles palabras de ánimo. La autoeficacia (Bandura, 1982) es el resultado de la acción pasada: el fracaso, la recaída en intentos anteriores, a menudo repetidos, menoscaba, comprensiblemente, la confianza en el éxito y, por lo tanto, la disposición a acometer un nuevo intento. Por supuesto, esta experiencia de esfuerzo y fracaso puede ser propia, tal como nos refieren muchos consultantes, pero, muy frecuentemente, es también vicaria: la observación o el conocimiento de la experiencia de fracaso de otros fumadores produce un efecto similar.

\section{La validación como estrategia definitoria de la entrevista motivacional}

La racionalidad del comportamiento adictivo y de las resistencias a su abandono es el fundamento de la estrategia de la aceptación o validación, elemento central y definitorio de la entrevista motivacional. 
Por 'validación' entendemos el conjunto de actuaciones que, durante la situación de consulta, se orienta a reconocer esa legitimidad y sentido y a manifestarla así al interlocutor. No hay entrevista motivacional, ni permeabilidad ante la influencia del profesional, si no hay aceptación o validación de la perspectiva del consultante. Esta aceptación hace posible que el consultante plantee abiertamente y pueda examinar sus discrepancias y ambivalencias, favoreciendo así la adopción de una decisión y un compromiso personales con el proceso.

Este concepto no es nuevo; por el contrario, tiene una larga y profunda raigambre en la ciencia del comportamiento. Miller y Rollnick $(1983,2015)$ proponen como principios estratégicos de la entrevista motivacional los propios de la psicoterapia de orientación fenomenológica ('terapia centrada en el cliente') del psicólogo norteamericano Carl Rogers. En su famoso artículo del año 1957 (Rogers, 1957), sin duda uno de los más influyentes de la historia de la psicología clínica, Rogers planteaba que existen tres condiciones necesarias y suficientes para que se produzca el cambio terapéutico: la congruencia o autenticidad por parte del profesional, la consideración positiva o aceptación de la perspectiva del consultante y la comprensión empática de esa perspectiva. Esta estrategia surge de un modelo según el cual el sufrimiento y la ambivalencia se deben a la incongruencia entre el yo tal como el individuo lo percibe y el conjunto de su experiencia como organismo, incongruencia que nace de la no aceptación de determinados aspectos de la experiencia, y especialmente determinados sentimientos y emociones, por ser inaceptables o no coherentes con la percepción del yo. Por lo tanto, el objetivo de la terapia es, según Rogers, conseguir que el yo sea congruente con la totalidad de la experiencia del consultante. La estrategia para conseguir esta congruencia es precisamente la aceptación incondicional (o validación) por parte del profesional, de todos los aspectos de la experiencia organísmica del consultante. Esta estrategia de validación será también el elemento más característico del tipo de relación entre profesional y consultante propio de la entrevista motivacional.

Obviamente, el fundamento de este principio estratégico es interpretable también desde la teoría del aprendizaje: si el comportamiento adictivo nace y se mantiene (o se modifica) en la transacción biográfica con los contextos, por distintos procesos de aprendizaje, como planteábamos en el epígrafe anterior, esto significa que nuestro comportamiento es el resultado de historias de aprendizaje únicas e irrepetibles, que dan lugar a patrimonios biográficos únicos e irrepetibles que se constituyen en la interacción con contextos y circunstancias únicas e irrepetibles. La situación clínica es un encuentro con una biografía que opera en un contexto. Considerar a un comportamiento (también el adictivo) como 'irracional' o 'patológico' es el resultado de no tomar en consideración el conjunto de experiencias, creencias, condicionamientos o repertorios de conducta que conforman en patrimonio biográfico de nuestro consultante, así como la función que el consumo cumple en la transacción con los contextos peculiares con los que interactúa (López y Costa, 2014).

\section{La entrevista motivacional es difícilmente compatible con un modelo de enfermedad}

El modelo del comportamiento adictivo como 'enfermedad crónica cerebral' es defendido, entre otros, por el National Institute on Drug Addiction (NIDA) norteamericano. El NIDA 
define la adicción como 'un trastorno médico que afecta al cerebro y modifica el comportamiento'. Un desarrollo detallado del mismo puede consultarse en varias publicaciones disponibles en la propia página web del NIDA $(2016,2018)$. Del mismo modo, es habitual dentro del campo médico definir la adicción al tabaco como una 'enfermedad crónica y recidivante'. La asunción básica de este modelo es que el comportamiento adictivo y la resistencia para su abandono serían síntomas de un trastorno en los circuitos cerebrales implicados en el sistema de recompensa, 'visibles' con técnicas de neuroimagen.

No es este el lugar para desarrollar las contradicciones del modelo, su inconsistente apoyo empírico, la evidencia a favor de un modelo contextualista, su endeblez epistemológica o lo lejos que está de ser universalmente admitido (véase, por ejemplo, González y Pérez (2007), López y Costa (2014), Pérez (2011), o la carta dirigida en 2014 por más de 80 expertos internacionales al editor de la revista Nature (2014) como crítica al modelo de enfermedad).

Lo que aquí nos interesa señalar es que, según lo expuesto, la estrategia de la validación, central y definitoria de la entrevista motivacional, es difícilmente compatible con un modelo de enfermedad. ¿Cómo validar y legitimar las resistencias, las dudas, las ambivalencias, los temores o las objeciones si los consideramos no como manifestación racional de la dificultad para abandonar un comportamiento profundamente enraizado que reporta importantes ventajas que compensan su mantenimiento, sino como síntomas de un trastorno, de una patología cuyo control escapa, por definición, al individuo que la padece? A menos, obviamente, que 'utilicemos' de modo postizo y ortopédico la entrevista motivacional del modo al que Rollick y Miller se refieren en la cita que abre este artículo, es decir, como un mero conjunto de técnicas o trucos sin atender a sus implicaciones conceptuales, como frecuentemente ocurre. Lo cierto es que el modelo de enfermedad es un escollo para la práctica efectiva de la entrevista motivacional, porque elude la consideración de la perspectiva del usuario y la necesidad de su participación activa. La entrevista motivacional reclama una visión contextualista, coherente con las terapias contextuales o de tercera generación (Pérez 2014), y que puede ser compatible (con matices) con las terapias cognitivo-conductuales.

Pensemos, además, que los principios y estrategias de la entrevista motivacional no son, como a menudo se tiende a asumir, algo que pone en juego antes de iniciar el proceso de intervención o tratamiento propiamente dicho, sino un estilo peculiar de intervención y de relación con el consultante, que se mantiene e impregna todo el proceso de la intervención, incluyendo, particularmente, los momentos de vacilación, duda, desánimo o recaídas en el consumo. Las recaídas o incumplimiento de propósitos, especialmente, son un momento importante para el enfoque de la entrevista motivacional porque a menudo implican una percepción de fracaso, con el consiguiente desánimo y riesgo de abandono, y su abordaje suele incluir un replanteamiento y, en su caso, renovación de la decisión de continuar, aprovechando la experiencia de aprendizaje que supone el episodio de recaída de cara al mantenimiento futuro de la abstinencia. No es fácil hacer compatible este enfoque con la visión fatalista de la recaída como un 'síntoma' de un 'trastorno crónico y recidivante'.

La estrategia de la validación, para ser coherente, supone un cambio en los roles que tradicionalmente se asumen en la relación entre profesional y usuario. Aquel ya no es un experto que dice al otro lo que hay que hacer, sino un colaborador en la tarea, que corresponde al 
consultante, de adoptar una decisión y de acometer, en su caso, un proceso de cambio. Esta relación de colaboración es difícilmente coherente con un modelo de enfermedad, en el que el paciente (no por casualidad se le denomina así) debe ponerse en manos del profesional, que asume un rol de experto que toma las decisiones sobre lo que en cada momento debe y no debe hacerse, dado que es el conocedor de la 'enfermedad' y del tratamiento adecuado.

\section{La disposición a dejar de fumar es contextual, y la entrevista motivacional no se fundamenta en un modelo de 'fases de cambio'}

Es sumamente frecuente encontrar referencias al conocido modelo de las fases de cambio de Prochaska y DiClemente (1982) como supuesto fundamento teórico de la estrategia de la entrevista motivacional. Habitualmente se asume que es preciso determinar en qué 'fase' del proceso se encuentra el consultante, con el fin de adecuar a ella los objetivos y la estrategia de la entrevista, asumiendo que la meta de la entrevista es hacer avanzar el proceso para pasar de una fase a otra, de modo que la actuación orientada al cambio efectivo solo es posible cuando el individuo se encuentra en la fase adecuada (preparación) para ello.

Esta vinculación es sorprendente por varios motivos, y, entre ellos, el hecho de que los propios Miller y Rollnick $(2009,2015)$ se han desmarcado de ella en más de una ocasión:

La teoría subyacente implícita de la entrevista motivacional solo ahora se está explicando, y no pretende ser una teoría integral del cambio. No es esencial ni importante utilizar las etapas del modelo transteórico cuando se aplica la entrevista motivacional. No es necesario asignar a las personas a una fase del proceso de cambio como parte de la intervención en entrevista motivacional.

En la primera de las publicaciones citadas, Miller y Rollnick atribuyen la confusión a dos cuestiones. Por un lado, a la coincidencia temporal en la presentación en sociedad de ambos modelos, a principios de la década de los 80 del siglo XX. Por otro, al hecho de que ambos llaman la atención sobre el mismo aspecto, sumamente relevante, y que constituye un avance considerable con respecto a la situación previa: existe una proporción elevada de sujetos con los que sería planteable un objetivo de cambio de comportamiento pero que no están dispuestos a tal cambio y con los que, por lo tanto, debe plantearse como objetivo previo la elaboración de la motivación o disposición para acometer ese cambio.

Tal vez, además, el éxito del modelo de las fases de cambio se deba a que se trata de una descripción sencilla y compatible con la generalizada visión cartesiana (o cognitivista) según la cual la motivación sería el resultado de la clarificación y consolidación de las ideas acerca de los beneficios del cambio, proceso que supuestamente exige unos plazos temporales, y también al hecho de que el modelo cumple la función de ofrecer una cierta referencia conceptual en un ámbito, el del cambio de conducta, en el que la mayoría de los profesionales proceden del campo biomédico y carecen de otros referentes teóricos.

Más allá de esto, se trata de dos modelos no solo independientes, sino, en buena medida, contrapuestos.

Así, el modelo de las fases del cambio describe (no explica) el cambio como resultado de una secuencia necesaria de etapas, de duración determinada, y en la que operan básicamente 
procesos de naturaleza cognitiva e interna en los que el peso condicionante de los aspectos del contexto no está claro. La implicación práctica, como queda dicho, es que no cabe iniciar el proceso de cambio hasta que el sujeto no se encuentra en la fase de preparación.

Ante todo, hay que señalar que la evidencia disponible está lejos de apoyar esta idea. La decisión de acometer un proceso de cambio, como dejar de fumar, no viene precedida necesariamente por una elaboración duradera por parte del sujeto de las ventajas y la importancia del cambio, sino que a menudo es disparada por aspectos del contexto. Entre ellos cabe citar la existencia de programas de deshabituación accesibles, la exposición de modelos, la aparición de una amenaza grave y repentina para la salud, el incremento de precios, campañas sensibilizadoras, cambios normativos que implican una limitación a la accesibilidad del tabaco o a la posibilidad de fumar... y, por supuesto, la habilidad de comunicación del profesional de la salud, con independencia de la supuesta 'fase' en la que previamente se encontrara el individuo. Y, lo que puede parecer más sorprendente, existe evidencia de que los resultados (abstinencia prolongada) no son peores entre quienes acometieron el proceso desde la llamada 'fase de precontemplación' que entre quienes lo hicieron desde la 'fase de preparación'. Así, en la revisión de la evidencia publicada por Riemsma et al. (2003) se concluye que las intervenciones dirigidas a la cesación tabáquica basadas en el modelo de las fases de cambio no son más efectivas que las no basadas en ese modelo. West y Sohal (2006) interrogaron a una muestra poblacional de fumadores y exfumadores y encontraron que en el $48 \%$ de los casos el último intento serio para dejar de fumar no había sido planificado previamente, es decir, no fue precedido de una fase previa de contemplación y, de modo claramente contradictorio con las predicciones del modelo de las fases, la proporción de intentos mantenidos al menos 6 meses fue mayor en los intentos no planificados $(65,4$ $\%$ vs. 42,3\%). Blanchard, Morgenstern, Morgan, Labouvie y Bux (2003) encontraron que ninguna medida de autoinforme de la motivación para el cambio, ni la que utiliza una escala continua ni la que utiliza un modelo de fases, resultó predictiva del cambio real. Carlson, Taerzer, Koopmans y Casebeer (2003), en una investigación sobre variables predictivas en un programa comunitario en grupos, encontraron que la variable 'fase' no añade valor predictivo a las variables demográficas o de historia del consumo. Tampoco en nuestros propios datos del programa de prevención y control del tabaquismo de Madrid Salud (Moreno 2006; Moreno y Herrero 2000) encontramos valor predictivo en la determinación de la 'fase' del proceso.

Frente a esta perspectiva, la entrevista motivacional se sustenta en una visión contextualista de la decisión y del cambio. Esto significa que la disposición a dejar de fumar surge y se consolida, en su caso, en las transacciones con los contextos con los que interactúa el fumador y, en el ámbito clínico, en la comunicación con el profesional de la salud como elemento significativo del contexto. Si no fuera así, si el profesional de la salud no tuviera la capacidad para influir en las decisiones de los consultantes, o esta fuera limitada por depender de la 'fase' previa en la que se encuentra el usuario, la entrevista motivacional no tendría sentido o tendría un alcance limitado, como a menudo parece asumirse.

Ciertamente, es sólida la evidencia sobre la efectividad del comportamiento del profesional en orden a favorecer la decisión de cambio en el consultante. Según señalan Miller y Rose 
(2009) en un esbozo de teoría del cambio (y también Apodaca y Longabaugh (2009) y Gaume, Gmel, Faouzi y Daeppen (2008)), los datos disponibles muestran que la probabilidad de iniciar un proceso de cambio de comportamiento está asociada de modo positivo y significativo con la magnitud del 'discurso del cambio' de parte del consultante durante la entrevista, la cual, a su vez, viene determinada por el comportamiento del profesional (contexto). Poulin, Button, Westra, Constantino y Antony (2018) encuentran que las medidas de autoinforme sobre motivación para el cambio no están asociadas a los resultados, y sí lo está el lenguaje automotivador durante la entrevista.

Así pues, el comportamiento del profesional y del consultante son interdependientes, de modo que la resistencia y la probabilidad de adoptar una decisión de cambio son fenómenos contextuales, no son internos o cognitivos ni dependen de la 'fase' de un proceso en la que se encuentre el consultante.

Fuera del ámbito clínico, esta vinculación de la disposición al cambio con desencadenantes o facilitadores del contexto es la justificación de la adopción de estrategias de salud pública dirigidas a aspectos del entorno que hagan más probable que los fumadores decidan abandonar su hábito, y que han demostrado eficacia, como las incluidas en los cambios legislativos todavía recientes en nuestro país.

Señalemos, por otra parte, que el modelo de las fases del cambio no puede sustentar la estrategia de la entrevista motivacional porque no es un modelo explicativo, sino únicamente descriptivo, del proceso. El modelo se denomina 'transteórico' precisamente porque se queda en una descripción de hechos (cuyo ajuste a la realidad es, como hemos visto, cuestionable), sin postular mecanismos explicativos, lo cual lo hace compatible con cualquier teoría. Un modelo explicativo, es decir, un modelo que vinculara el fenómeno explicado con una serie de variables que afectaran a su probabilidad, no sería en modo alguno 'transteórico'. La pretendida virtud de ser compatible con distintas teorías limita su alcance. Obviamente, un modelo debe servir para orientar la acción práctica y debe, por lo tanto, especificar las variables sobre las que se ha de intervenir. Es cierto que el modelo de las fases hace referencia también a procesos ('aumento de conciencia', 'autorreevaluación', 'reevaluación ambiental', 'liberación social', 'contracondicionamiento' y otros) pero es un aspecto notablemente menos desarrollado y sustancial, prácticamente ignorado en las versiones más difundidas del modelo y que no guardan relación con las estrategias que se ponen en juego durante la entrevista motivacional. Callaghan y Herzog (2006), además, no encuentran asociación entre la transición de la fase de contemplación a la fase de preparación y el uso de procesos postulados por el modelo, poniendo en cuestión que esos procesos constituyan los mecanismos responsables de la transición entre fases. Rosen (2000), a partir de un metaanálisis sobre 47 estudios que abordan los procesos de cambio en distintas conductas relacionadas con la salud, concluye que en el paso de la precontemplación a la contemplación intervienen procesos de cambio en la interacción con el contexto, como cambios de conducta, control estimular o uso de reforzadores.

Pero lo más relevante de esta extraña pero habitual asociación de la entrevista motivacional con los postulados de modelo de las fases de cambio es, sin duda, el efecto práctico que produce: se asume que, dado que el proceso del cambio sigue de modo fijo una secuencia 
de fases, los objetivos y las estrategias del consultor deben adecuarse a la fase en la que se encuentra el consultante, resultando inútil y contraproducente pretender, en particular, que un fumador al que se sitúa en fase de 'precontemplación' adopte en la misma sesión o en un plazo breve una decisión de abandono del consumo de tabaco, y menos aún que dé los primeros pasos efectivos en esa dirección, suponiendo que lo más que puede hacerse es contribuir a que avance el proceso y que el usuario vaya ‘elaborando' su decisión. Aunque, ciertamente, el modelo de las fases del cambio ha sido matizado desde sus primeras formulaciones, asumir sus postulados funciona, en numerosas ocasiones, como un corsé que limita el alcance y la efectividad de la entrevista motivacional. Existe el riesgo de que la actuación se quede en palabras y poco más. Señalemos, de paso, que son frecuentes las situaciones clínicas en las que el cambio de comportamiento es urgente y no puede demorarse hasta que el usuario llegue a la fase adecuada porque se pondría en riesgo su salud o su vida, como la del fumador con grave patología cardiovascular asociada, la del joven bebedor excesivo que conduce su coche bajo los efectos del alcohol cada fin de semana o la de la joven que mantiene de modo habitual relaciones sexuales sin protección.

\section{La motivación se construye en la acción}

Otra insuficiencia detectable en la formación y en la práctica habitual de la entrevista motivacional, nacida igualmente del mismo sesgo cognitivista, tiene que ver con la relación entre la disposición al cambio y la acción.

La visión cartesiana que rige la práctica habitual de la entrevista motivacional asume que la motivación es previa y desencadenante de la acción, y se construye a través de un proceso esencialmente cognitivo ('reflexión', 'cambio de actitud', 'toma de conciencia'...). Hay, pues, que motivarse primero para actuar después. En términos del modelo de las fases de Prochaska y DiClemente, diríamos que hay que estar en la fase de preparación para pasar a la acción, que es la fase siguiente. Se trata de una visión compartida, en su esencia, por las terapias cognitivas: cambiar las ideas para cambiar las acciones. La relación entre motivación y acción podría representarse gráficamente del modo siguiente:

Este modelo parece olvidar que, tal como establece el paradigma de condicionamiento instrumental, el principal motor de la disposición para practicar un determinado comportamiento es precisamente la experiencia, directa o vicaria, de la relación entre la acción y sus resultados, en la medida en que a través de la acción obtenemos determinados efectos (refuerzo) y comprobamos también nuestra capacidad para llevarla a cabo (percepción de autoeficacia), lo cual incrementa o disminuye la disposición a seguir actuando del mismo modo. Así pues, la motivación se construye en la acción, tal como representamos en la siguiente figura.

Figura 1.- La motivación como estado previo y desencadenante de la acción.

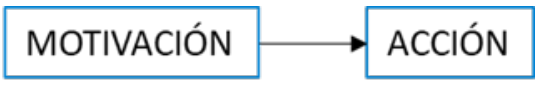


Este principio es fácil de identificar en nuestra experiencia cotidiana. Las acciones nuevas que emprendemos en nuestra vida con frecuencia no son desencadenadas por un estado de disposición previa, sino por elementos que aparecen en el contexto y que nos predisponen a ellas, nos las facilitan o nos obligan a ponerlas en práctica. Es más probable que dejemos de fumar si un cambio normativo prohíbe fumar en el lugar de trabajo. Empezamos a estudiar un primer idioma extranjero porque se nos impone en el programa de asignaturas de la escuela y es después, en la medida en que adquirimos cierto dominio y obtenemos resultados de su práctica, cuando decidimos seguir estudiándolo y practicándolo.

Figura 2.- La motivación como resultado de la acción

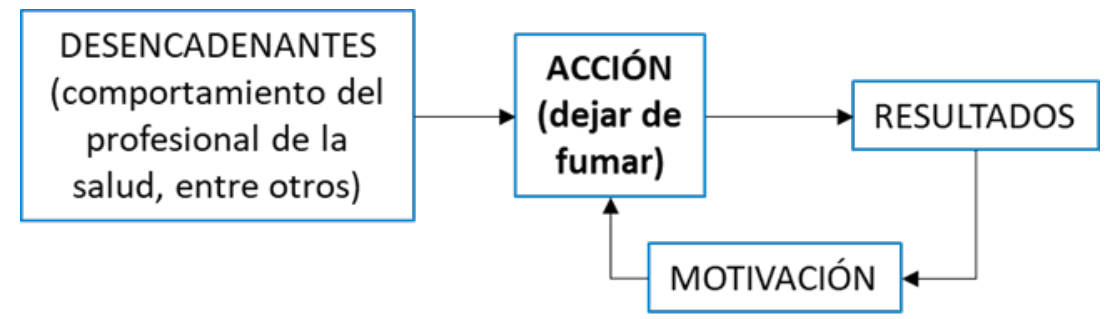

La aplicación clínica de este principio estratégico es central en las terapias conductuales de tercera generación (Pérez, 2014) y, de modo particular, en la terapia de activación conductual para la depresión (Kanter, Busch y Rusch, 2011; Martell, Addis y Jacobson, 2001). En el caso de la entrevista motivacional, la implicación práctica consiste en invitar al consultante a actuar, a hacer la prueba, a llevar a cabo pequeños experimentos como forma de remover obstáculos y favorecer la toma de decisiones. Apresurémonos a insistir en que esta invitación a la acción puede no ser sencilla y requiere, para ser efectiva, de ciertas condiciones previas que aquí nos limitaremos a citar: un contexto interpersonal basado en la validación de las dudas y ambivalencias del consultante, el uso de recursos de comunicación (metáforas o analogías, por ejemplo) que hagan ver la importancia de la acción como forma de reducir la ambivalencia, y acciones específicas orientadas a identificar y remover obstáculos para la acción (técnicas de control estimular, elaboración de un plan, división de la tarea en objetivos alcanzables, aprendizaje de habilidades, información sobre recursos, provisión de reforzadores efectivos, etc.).

\section{Conclusiones}

Las consideraciones críticas que planteamos en torno a la práctica habitual de la entrevista motivacional pueden resumirse en la frecuente e incoherente cohabitación de esta práctica con dos conceptos: el modelo psicopatológico o de enfermedad, particularmente en el campo de las conductas adictivas, y el conocido modelo transteórico de las 'fases de cambio'.

Por lo que se refiere al primer punto, excedería con mucho el espacio disponible hacer referencia a su inconsistencia epistemológica, a su endeble apoyo empírico o a las contradicciones con las prácticas que el mismo modelo propugna. Nos limitamos aquí a señalar la 
contradicción entre un enfoque esencialmente invalidante de la perspectiva del consultante (el etiquetaje psicopatológico y la consiguiente sumisión al rol de experto que asume el profesional) y la práctica de la validación de esa perspectiva como estrategia central y definitoria de la entrevista motivacional. Esta situación se asocia a una práctica ortopédica que limita no solo su coherencia, sino también su efectividad.

En cuanto a la supuesta vinculación de la práctica de la entrevista motivacional con el modelo de las 'fases de cambio', defendemos frente a ella una visión contextualista del comportamiento y del cambio, que implicaría importantes ventajas prácticas. En primer lugar, la incorporación, en la entrevista, de la acción misma como estrategia que construye, de modo potente, la disposición al cambio, en la medida en que la motivación es el resultado de la relación entre la acción y los resultados (condicionamiento instrumental). Por otro lado, la posibilidad del paso a la acción con independencia de la supuesta 'fase' en la que se encuentre el consultante, evitando demorar ese paso hasta que el consultante se encuentre 'preparado' tras un proceso esencialmente cognitivo, una demora que, además de injustificada teóricamente, no es deseable y no siempre es posible en la situación clínica.

\section{REFERENGIAS}

Apodaca, T. R.; Longabaugh, R. (2009). Mechanisms of change in motivational interviewing: a review and preliminary evaluation of the evidence. Addiction, 104, 705-715.

Bandura, A. (1982). Teoría del Aprendizaje Social. Madrid: Espasa-Calpe.

Becker, J.; Hungerbuehler, I.; Berg, 0.; Szamrovicz, M.; Haubensack, A.; Kormann, A.; Schaub, M.P. (2013). Development of an integrative cessation program for co-smokers of cigarettes and cannabis: demand analysis, program description, and acceptability. Substance Abuse Treatment, Prevention, and Policy 8, 33-45.

Blanchard, K. A.; Morgenstern, J.; Morgan, T. J.; Labouvie, E.; Bux, D. A. (2003). Motivational subtypes and continous measures of readiness for change: concurrent and predictive validity. Psychology of Addictive Behaviors, $17,1$.

Callaghan, R. C.; Herzog, T. A. (2006). The relation between processes of change and stage-transition in smoking behaviour: a two-year longitudinal test of the Transtheorical Model. Addictive Behaviors 31 (8), 1331-45.

Carlson, L. E.; Taerzer, P.; Koopmans, J.; Casebeer, A. (2003). Predictive value of aspects of the Transtheoretical Model on smoking cessation in a community-based, large-group cognitive behavioral program. Addictive Behaviors 28.

Comité Nacional para la Prevención del Tabaquismo (2015). Proyecto EVICT. Informe 2015. Disponible en http:// evictproject.org/wp-content/uploads/2015/09/Informe_EVICT_2015.pdf

Costa M. y López E. (2008). Educación para la Salud. Guía práctica para promover estilos de vida saludables. Madrid: Pirámide.

Costa, M. y Moreno, J. J. (2008) ¡Dispuestos a dejar de fumar! Cómo hacerlo fácil. Una guía para el consejo clínico. Madrid Salud. Disponible en http://www.madridsalud.es/pdf/publicaciones/saludpublica/GuiaDEJARdeFUMAR.pdf

Davis, M. L.; Powers, M. B.; Handelsman, P.; Medina, J. L.; Zvolensky, M.; Smits, J. A. (2015) Behavioral Therapies for Treatment-Seeking Cannabis Users: A Meta-Analysis of Randomized Controlled Trials. Evaluation \& the Health Professions 38/1) 94-114.

Gates, P. J.; Sabioni, P.; Copeland, J.; Le Foll, B.; Gowing, L. (2016). Psychosocial interventions for cannabis use disorder. Cochrane Database of Systematic Reviews 2016, Issue 5, art. No. CD005336. 
Gaume, J.; Gmel, G.; Faouzi, M.; Daeppen, J. B. (2008). Counsellor behaviours and patient language during brief motivational interventions: a sequential analysis of speech. Addiction, 103, 1793-1800.

González, H. y Pérez, M. (2007). La invención de trastornos mentales. Madrid: Alianza.

Hettema, J.; Steele, J.; Miller, W.R. (2008). Entrevista Motivacional. RET, Revista de Toxicomanías, 52.

Kanter, J.W.; Busch, A.M.; Rusch, L.C. (2011). Activación Conductual. Refuerzos positivos ante la depresión. Madrid: Alianza.

Lee, D. C.; Budney, A. J.; Brunette, M. F.; Hughes, J. R.; Etter, J. F.; Stanger, C. (2014). Treatment models for targeting tobacco use during treatment for cannabis use disorder: Case series. Addictive Behaviors 39, 1224-1230

López, E. y Costa, M. (2014). Los problemas psicológicos no son enfermedades. Una crítica radical de la psicopatología. Madrid: Pirámide.

Lundahl, B.W.; Kunz, C.; Brownell, C.; Tollefson, D.; Burke, B. L. (2010). A meta-analysis of Motivational Interviewing: twenty-five years of empirical studies. Research on Social Work Practice 20.

Martell, C. R.; Addis, M. E.; Jacobson, N. S. (2001). Depression in context: Strategies for guided action. Nueva York: W. W. Norton.

Miller, W. R. (1983). Motivational interviewing with problem drinkers. Behavioural Psychotherapy, 11, 147-172

Miller, W. R.; Rollnick, S. (2009). Ten things that Motivational Interviewing is not. Behavioural and Cognitive Psychotherapy, 37, 129-140.

Miller, W. R.; Rollnick, S. (2015). La entrevista motivacional. Ayudar a las personas a cambiar (3ª ed.). Barcelona: Paidos.

Miller, W. R.; Rose, G. S. (2009). Toward a theory of motivational interviewing. American Psychologist 64, 6, 527-537.

Moreno, J. J. (2006). El programa para dejar de fumar "on line" del Ayuntamiento de Madrid. Un estudio exploratorio. Adicciones, 18, 4.

Moreno, J. J.; Herrero, F. J. (2000). Factores asociados al éxito en programas para dejar de fumar. Adicciones, $12,3$.

National Institute on Drug Abuse (NIDA) (2016). Entendiendo el uso de drogas y la adicción. Disponible en https:// www.drugabuse.gov/es/publicaciones/drugfacts/entendiendo-el-uso-de-drogas-y-la-adiccion

National Institute on Drug Abuse (NIDA) (2018). Las drogas, el cerebro y la conducta: la ciencia de la adicción. Disponible en https://www.drugabuse.gov/es/publicaciones/serie-de-reportes/las-drogas-el-cerebro-y-el-comportamiento-la-ciencia-de-la-adiccion/prefacio

Nature (2014). Letter to the editor of Nature. Nature 506.

Pérez, M. (2011). El mito del cerebro creador. Madrid: Alianza.

Pérez, M. (2014). Las terapias de tercera generación como terapias contextuales. Madrid: Síntesis.

Poulin, L. E.; Button, M. L.; Westra, H. A.; Constantino, M. J.; Antony, M. M. (2018). The predictive capacity of self-reported motivation vs. early observed motivational language in cognitive behavioural therapy for generalized anxiety disorder. Cogn Behav Ther 21, 1-16.

Prochaska, J. 0.; DiClemente, C. C. (1982). Transtheoretical therapy: toward a more integrative model of change. Psychotherapy: theory, research and practice 19, 276-288.

Riemsma, R. P.; Pattenden, J.; Bridle, C.; Sowden, A. J.; Mather, L.; Watt, I. S.; Walker, A. (2003). Systematic review of the effectiveness of stage based interventions to promote smoking cessation. BMJ 326, 1175-1177

Rollnick, S.;Miller, WR. (1996) ¿Qué es la Entrevista Motivacional? Revista española de toxicomanías 6; 3

Rogers, C. R. (1957). The necessary and sufficient conditions of therapeutic personality change. Journal of Consulting Psychology 21, 95-103.

Rosen, C. S. (2000). Is the sequencing of change processes by stage consistent across health problems? A meta-analysis. Health Psychol. 19 (6), 593-604.

West, R.; Sohal, T. (2006). Catastrophic pathways to smoking cessation: findings from national survey. BMJ 332, 458-60. 